Sādhanā Vol. 40, Part 3, May 2015, pp. 769-785. (C) Indian Academy of Sciences

\title{
Suppression of vortex shedding around a square cylinder using blowing
}

\author{
ARUN K SAHA* and ANKIT SHRIVASTAVA \\ Department of Mechanical Engineering, Indian Institute of Technology Kanpur, \\ Kanpur-208016, India \\ e-mail: aksaha@iitk.ac.in; ashri@iitk.ac.in
}

MS received 13 June 2014; revised 12 October 2014; accepted 20 November 2014

\begin{abstract}
Direct numerical simulation (DNS) of flow past a square cylinder at a Reynolds number of 100 has been carried out to explore the effect of blowing in the form of jet(s) on vortex shedding. Higher order spatial as well as temporal discretization has been employed for the discretization of governing equations. The varying number of jets, jet velocity profiles and different blowing velocities are studied to investigate the characteristics of vortex shedding. The parabolic velocity profile has been found to be more effective in suppressing the vortex shedding as compared to the uniform velocity. Complete suppression of vortex shedding along with remarkable reduction in drag coefficient has been achieved for both jet velocity profiles but at different velocities. The corresponding values for uniform and parabolic jet profiles are 0.87 and 0.6 , respectively at a mass flux of 0.120 . The study also reveals that there is considerable effect of the number of jets on the vortex shedding phenomena.
\end{abstract}

Keywords. Square cylinder; vortex shedding; jets; blowing; suppression.

\section{Introduction}

The bluff bodies like circular cylinders, rectangular prisms, etc. are the most common engineering structural configurations resembling buildings, bridges, chimneys, cooling towers, etc. Engineers quite often face various flow-induced difficulties, in dealing with flow around these bodies, most common of which include the flow-induced vibrations. For a square cylinder kept in a uniform flow at very low Reynolds number below a value of 1.16, the flow remains laminar, two-dimensional, and steady and does not separate as reported by Sen et al (2011). Lankadasu \& Vengadesan (2008) showed that as the Reynolds number is increased further, the flow starts to separate and but remains steady and two-dimensional up to a Reynolds number of 47 . The flow becomes unstable and shows unsteadiness for Reynolds number greater than 47 . With an increase of Reynolds number beyond 161 (Saha 2013), the flow exhibits three-dimensionality though the shedding remains periodic and laminar. Because of unsteady nature of the flow at

*For correspondence 
a relatively higher Reynolds number, significant drag and lift fluctuations, acoustic noise and large structural vibrations are encountered. If the natural frequency of the structure and flowinduced oscillation frequency matches, it can lead to catastrophic structural failure. This calls for an in-depth study to control the wake dynamics so that such structural damage can be avoided.

Flow control methods have broadly been classified as (a) active and (b) passive methods. The methods that require expenditure of energy from external sources for flow control are classified as active methods and those in form of surface modification or additional attachment that manage to modify the flow without the expenditure of energy are referred to as passive methods. A variety of passive methods that use the surface modifications are roughness, dimples (Choi 2006), helical strakes (Lee \& Kim 1997), longitudinal grooves, splitter plate (Kwon \& Choi 1996), small secondary control cylinders (Strykowski \& Sreenivansan 1990). Raghavan et al (2008) provided a very comprehensive overview of all the passive methods used to control the vortex-induced vibrations. Equivalently, there are enormous number of active methods found to cater to the needs of flow control such as stream-wise or transverse oscillations of bluff body (Blackburn \& Henderson 1999; Cetiner \& Rockwell 2003), wall heating of the cylinder, modifying the local fluid properties (Gad-el-Haq 2000), steady blowing or suction (Fransson et al 2004), electromagnetic forcing (Kim \& Lee 2000), periodic blowing or suction (Biringen 1984) and external excitation through actuators. Zdravkovich (1981) worked on the various methods of flow namely, (i) surface protrusions affecting separated shear layers, (ii) shrouds affecting entrainment layers and (iii) near wake stabilizers preventing shear layer interaction. Choi et al (2008) provided a condensed overview of all the flow control methods for the suppression of vortex shedding past a circular cylinder.

In spite of the large number of passive methods available, the use of active methods has received less emphasis. Furthermore, among all the active methods, considerable research work has been concentrated on using the methods of blowing and suction due to the simplicity in its working mechanism and flexibility in its implementation. All methods using suction or blowing utilize the two fundamental concepts in controlling the vortex formation and its subsequent shedding as explained by Gerrard (1966). He explained that the formation of unsteady wake depends on the conditions namely, (i) shear layers should roll up to form vortex of 'sufficient' strength and (ii) the two shear layers must interact with each other within a critical stream-wise distance to become unstable. Blowing or suction disrupt either of the above necessary conditions and helps in suppressing the vortex shedding.

Mathelin et al (2002) used a numerical approach to study the effect of blowing on heat transfer and flow around a porous circular cylinder for Reynolds number range of 10-7000 and could show a consistent decrease in the Strouhal Number with increasing blowing velocity. The pioneering experimental work of Wood (1967) on the effect of base bleed on wakes has given a deeper insight into the subject of control of vortex shedding. He used a two-dimensional brass aerofoil like model with elliptic nose and a parallel rear section with open rear end. His result of the visualizations on a two-dimensional brass aerofoil model confirms that the base bleed leads to an increase in formation region and elongated shear layers. Consequently, large magnitude of vorticity carried by the shear layer is dissipated even before they grow to full grown vortices. This leads to formation of vortices having strength which is insufficient to cause vortex shedding, which is well in concurrence with the basic principles of vortex shedding suggested by Gerrard (1966). Also, Cohen (1991) through the use of scaling arguments put forward an analytical model for predicting Strouhal frequency in flow around porous circular cylinders undergoing uniform blowing or suction. His important findings include that for $\operatorname{Re}_{\mathrm{o}}^{2} / \operatorname{Re}_{\infty} \gg 1\left(\operatorname{Re}_{\mathrm{o}}\right.$ denotes the Reynolds number in terms of blowing/suction velocity, $\operatorname{Re}_{\infty}$ 
denotes the Reynolds number of flow at far field), the control becomes effective otherwise inspite of blowing/suction, the cylinder behaves as an impervious body. He predicted a uniform increase in Strouhal number with suction velocity but it was later contradicted by several other numerical and experimental studies (Mei 1992) where it has been demonstrated that the increase is possible only for suction velocity below a critical value. Dong et al (2008) addressed the issue of effectiveness of blowing or suction on suppression of shedding around a circular cylinder at $\operatorname{Re}=500$ and 1000 through the computations of the three-dimensional unsteady Navier-Stokes equations and came up with a novel method which they called the 'WSLB'-Windward Suction and Leeward Blowing method. Their findings suggest that 'suction-only' is effective only in reducing lift if it is operated at very high velocities while low velocities tend to have the opposite effect.

Most of the work focuses on the effect of uniform blowing or suction on vortex shedding around circular cylinders. There are a few studies in literature available which investigate the control of vortex shedding of square cylinders using blowing or suction. Fransson et al (2004) experimentally studied the flow around a porous square cylinder subject to uniform blowing and suction at a Reynolds number of $10^{4}$ and concluded that suction delayed separation leading to a narrower wake width while blowing exhibited an opposite behaviour altogether. They found that the Strouhal number increases by $50 \%$ in case of suction and decreases by $25 \%$ with use of blowing. Their investigations showed that the drag coefficient increases linearly with increasing blowing rate but decreases steeply (almost $70 \%$ reduction) at a particular suction velocity. Arcas \& Redekopp (2004) performed numerical simulations to study the effect of 'base' blowing or suction on vortex shedding from a plane fore-body with a rectangular base placed within a channel. Two symmetrically placed blowing slots of varying size were used with different velocities. They found that the bleed flux $\left(=v_{\mathrm{w}} \mathrm{B}\right.$ where $v_{\mathrm{w}}$ is blowing/suction velocity and $\mathrm{B}$ is slot thickness) required for control becomes asymptotic with increasing Reynolds number. Furthermore, they showed that suction seemed to be quite sensitive to both the flux distribution and its magnitude. Cuhadaroglu et al (2007) studied experimentally the influence of uniform injection through one perforated surface of a square cylinder on pressure and drag coefficient in turbulent flow regime $\left(\operatorname{Re}=10^{4}, 1.6 \times 10^{4}, 2.4 \times 10^{4}\right)$. Their results pointed out that the drag coefficient increases with injection rate when the opening is placed at the front or top face but decreases modestly when injection is done from rear face.

Kim et al (2003) carried out a parametric study in order to investigate the effect of base jet on vortex shedding both in laminar $(\operatorname{Re}=200)$ and turbulent $(\operatorname{Re}=8520)$ flow around a square cylinder placed symmetrically inside a channel. They were able to find out the optimum injection ratio at which the lift coefficient fluctuation was found to be minimum with a significant change in vortex formation. Akansu \& Firhat (2010) investigated experimentally the flow around a square prism having injection from its base at a Reynolds number of 8000 . The injection ratios at which experiment was carried out were chosen to be 1.12, 1.68, 2.24 and 2.8. They observed two different regimes in the wake of the cylinder with a change in injection velocity. In first case, at low injection ratio, the vortex are displaced a bit downstream decreasing the shedding frequency while in second case, at very high injection ratio, jet penetrates into the vortices shifting formation region remarkably downstream. Consequently, weaker separated shear layers are formed resulting in a thinner width wake and thus causes the shedding frequency to increase. Mei (1992) in her extensive work on Strouhal frequencies for flow past square cylinder with uniform surface suction or blowing through all four sides found that for suction velocity between 0.025 and 0.1 , the Strouhal frequency has been found to increase. She also showed complete suppression of vortex shedding if suction velocity falls between 0.40 and 
0.45. A total suppression could also be achieved for blowing velocities ranging between 0.15 and 0.20 .

As quite evident from the studies cited above, most of the work that concentrates on square cylinders is focused on 'uniform' blowing/suction' on 'porous/perforated' cylinders and has no mention of the effect of number of jets, their positions and jet velocity distribution on the wake behind the cylinder. Also most of them illustrated the suppression of vortex shedding without any mention of complete suppression. The present study along with achieving complete suppression with single jet at rear face of the cylinder also elucidates the effect of number of jets and effect of the velocity profile of the blowing jet on vortex shedding. In addition, the present study also discusses the effect of blowing on the integral parameters.

\section{Problem statement}

A square cylinder of side D is kept in an infinite medium having a cross flow. A single Reynolds number (based on the cylinder dimension and the average velocity at inlet) of 100 is considered in the present numerical study. The two-dimensional geometrical model of the problem has been depicted in figure 1 . The origin of the coordinate system coincides with the center of the cylinder. The dimensions related to the geometry are $\mathrm{H}=20 \mathrm{D}$ thus giving a blockage $(\mathrm{D} / \mathrm{H})$ of $5 \%, \mathrm{~L}_{\mathrm{u}}=$ 7.0D; $\mathrm{L}_{\mathrm{d}}=20 \mathrm{D}$.

To achieve the overall objectives, the analysis has been carried out by addressing the problem as three small sub-problems:

(a) Problem-I: The effect of blowing through a single jet at the rear face on the vortex shedding phenomena has been studied. The velocity profile at the jet exit is chosen to be uniform for this analysis.

(b) Problem-II: The second problem addresses the effect of velocity profile at jet exit on the vortex shedding. Two different types of velocity profile namely, uniform and parabolic velocity profiles are chosen.

(c) Problem-III: Finally, the effect of number of jets having parabolic jet profile on the vortex shedding has been addressed. The geometrical models for various arrangements are shown in figure 2.

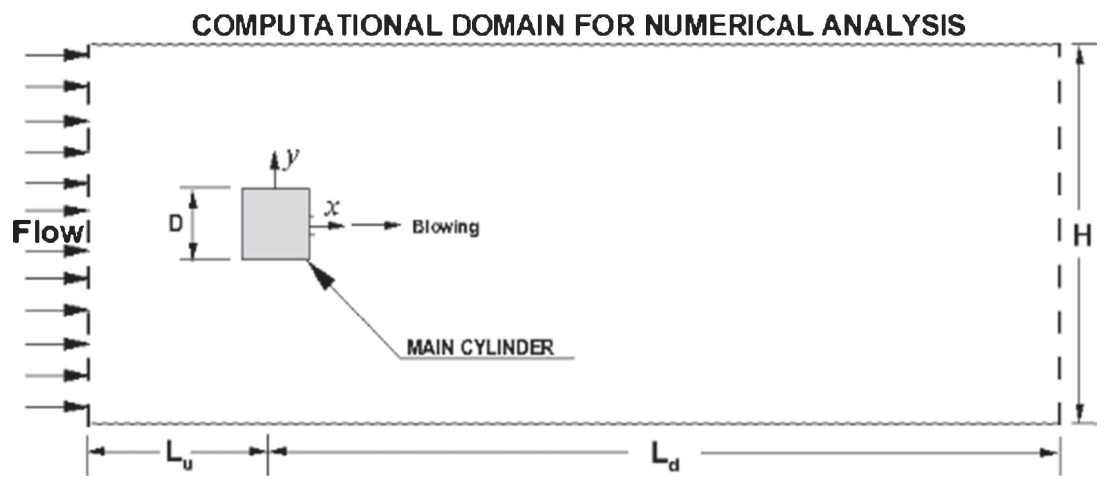

Figure 1. Two-dimensional computational domain. 


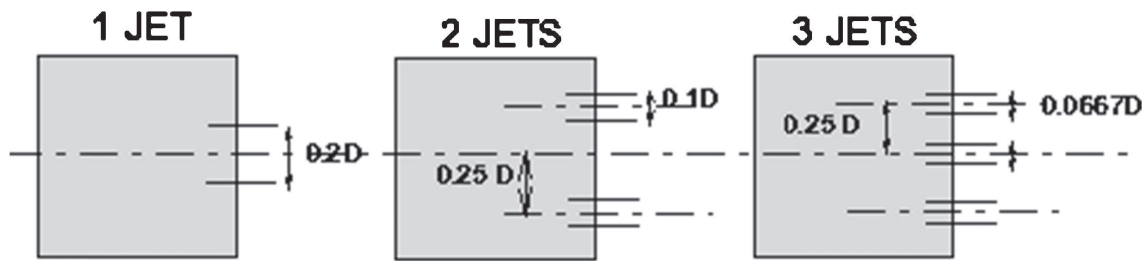

Figure 2. Arrangement of jets on cylinders with one-jet, two-jets and three-jets in problem-III.

\section{Mathematical formulation}

\subsection{Governing equations}

The computation of flow field around the square cylinder is carried out by solving twodimensional unsteady, Navier-Stokes equations for incompressible fluids. The governing equations of continuity and momentum in $\mathrm{x}$ - and $\mathrm{y}$-directions are represented as follows in Eqs. (1), (2), and (3), respectively:

$$
\begin{gathered}
\frac{\partial u}{\partial x}+\frac{\partial v}{\partial y}=0 \\
\frac{\partial u}{\partial t}+\frac{\partial(u u)}{\partial x}+\frac{\partial(u v)}{\partial y}=-\frac{\partial p}{\partial x}+\frac{1}{R e}\left(\frac{\partial^{2} u}{\partial x^{2}}+\frac{\partial^{2} u}{\partial y^{2}}\right) \\
\frac{\partial v}{\partial t}+\frac{\partial(u v)}{\partial x}+\frac{\partial(v v)}{\partial y}=-\frac{\partial p}{\partial y}+\frac{1}{R e}\left(\frac{\partial^{2} v}{\partial x^{2}}+\frac{\partial^{2} v}{\partial y^{2}}\right) .
\end{gathered}
$$

The above equations are non-dimensionalized using with the average velocity $\mathrm{u}_{\infty}$ at the inlet, all lengths with the obstacle height $\mathrm{D}$ and the pressure using $\rho \mathrm{u}_{\infty}^{2}$.

\subsection{Boundary conditions}

The boundary conditions employed for the present investigations are as mentioned below.

Inflow: A uniform velocity has been prescribed

$$
u(y)=U_{\infty}=1.0, v=0 .
$$

Outflow: To this end, the convective boundary conditions proposed by Orlanski (1976) have been used, which does not affect the flow in the upstream.

$$
\frac{\partial \emptyset}{\partial \mathrm{t}}+\mathrm{u}_{\mathrm{c}} \frac{\partial \emptyset}{\partial \mathrm{x}}=0
$$

where $\emptyset=u, v$

Transverse confining surfaces: The confining boundaries (top and bottom boundaries) are modelled as the free slip boundaries

$$
\text { For } y= \pm \frac{\mathrm{H}}{2}, \quad \frac{\partial \mathrm{u}}{\partial \mathrm{y}}=0, v=0 .
$$


Obstacle: No-slip $(\mathrm{u}=\mathrm{v}=0)$ boundary conditions are used for the velocities on the main cylinder surfaces. However, at the jet locations, the normal velocity is prescribed with zero tangential velocity.

Jet outlet: The jet velocity $V_{j}$ is set to various velocity or velocity ratio $\left(V_{j} / U_{\infty}\right)$ which is equal to jet velocity as $\mathrm{U}_{\infty}=1.0$.

\section{Solution methodology}

The equations as mentioned from Eqs. (1)-(3) are discretized using finite difference method on a non-uniform staggered grid. The technique employed is an improved version by Harlow \& Welch (1965) called the MAC (Marker and Cell) method. When the flow is incompressible, pressure and velocity are to be solved simultaneously such that the pressure field is compatible with the continuity equation. This is taken care of by using two steps procedure. In the first step, provisional values of velocity components are explicitly calculated using previous time step values. However, these values thus obtained need not necessarily satisfy the continuity equation. So, in the second step the pressure and velocity components are corrected using the pressure correction equations such that mass balance (continuity) is satisfied simultaneously. An explicit, second order Adams-Bashforth scheme is used for time advancement of convection and diffusion terms. Furthermore, the convection and diffusion terms have both been approximated by second-order central differencing scheme.

Mathematically, the method can be expressed as follows.

The momentum equation is written using a space operator $g$, which contains the convection as well as the diffusion terms as

$$
\frac{\partial u_{i}}{\partial t}=\mathrm{g}\left(u_{i}, u_{j}\right)-\frac{\partial p}{\partial x_{i}} .
$$

The predictor step for the time advancement takes the form

$$
\frac{u_{i}^{*}-u_{i}^{n}}{\Delta t}=\frac{3}{2} \mathrm{~g}\left(u_{i}, u_{j}\right)^{n}-\frac{1}{2} \mathrm{~g}\left(u_{i}, u_{j}\right)^{n-1}-\frac{\partial p^{n}}{\partial x_{i}} .
$$

This gets followed by the corrector step

$$
p^{\prime}=-\frac{r_{o} \frac{\partial u_{i}^{*}}{\partial x_{i}}}{\left[2(\Delta t)\left\{\frac{1}{(\Delta x)^{2}}+\frac{1}{(\Delta y)^{2}}\right\}\right]} .
$$

The final solution for velocity and pressure are given as Eqs. (7) and (8), respectively

$$
\begin{gathered}
u_{i}^{n+1} \leftarrow u_{i}^{*}+\frac{\Delta t}{\Delta x_{i}} p^{\prime} \\
p^{n+1} \leftarrow p^{n}+p^{\prime} .
\end{gathered}
$$

The corrector steps Eqs. (6)-(8) is solved using Gauss-Siedel iterations with $r_{0}$ as the over relaxation factor $(=1.8)$ to accelerate the pressure correction process. The solution method has been discussed in greater detail in Saha (2013).

For, computation the flow domain is divided into numerous rectangular cells. The grid is non-uniform in both the directions and is clustered near the walls of the obstacle to resolve the 
boundary layers. The minimum grid size used in the present study is 0.005 . In addition to fine grids near the walls of the obstacle, the sufficient grids with adequate resolution is also for the jets. The number of grids used for the baseline case without any jets was $355 \times 264$. The typical grid size used for one-jet, two-jets, three-jets and four-jets cases are $346 \times 294,346 \times 304$, $346 \times 309$ and $346 \times 316$, respectively. A typical grid for the case with one jet is shown in figure 3 .

The simulations for all cases were started using quiescent initial conditions $(u=v=0$; $p=1.0$ ). The dependency of the solutions on the initial conditions has also been checked by taking another conditions $(u=1.0, v=0 ; p=1.0)$ and no differences have been observed in the dynamic steady state results of the two simulations. The unsteady simulations were also tested for time-step size independence. The time-dependent simulations were found to show insignificant variations in Strouhal number and drag forces. The present code has been validated thoroughly for the flow past a square cylinder by comparing the results with available numerical as well as experimental results in the Reynolds number range of 100-400 (Saha et al 2000).

The dependency of the domain size on the flow field is also undertaken by taking two different domain sizes $\left(\mathrm{L}\left(=\mathrm{L}_{\mathrm{u}}+\mathrm{L}_{\mathrm{d}}\right) \times \mathrm{H}\right)$ namely, $27 \times 20$ and $32 \times 24$. The differences in drag coefficient and Strouhal for the two cases are seen to be negligible.

The grid independence study has been undertaken using two different grid sizes. For the study, a coarse grid $279 \times 232$ roughly 1.5 times less than the original grid $346 \times 294$ was used in the case of a single jet. With both grids, a complete suppression was achieved. The instantaneous flow structures are also found to be identical for results generated using two grid sizes The drag coefficient is also found match for both the grids. Therefore, the results with $346 \times 294$ grid points or scaled grids for other cases are grid independent.
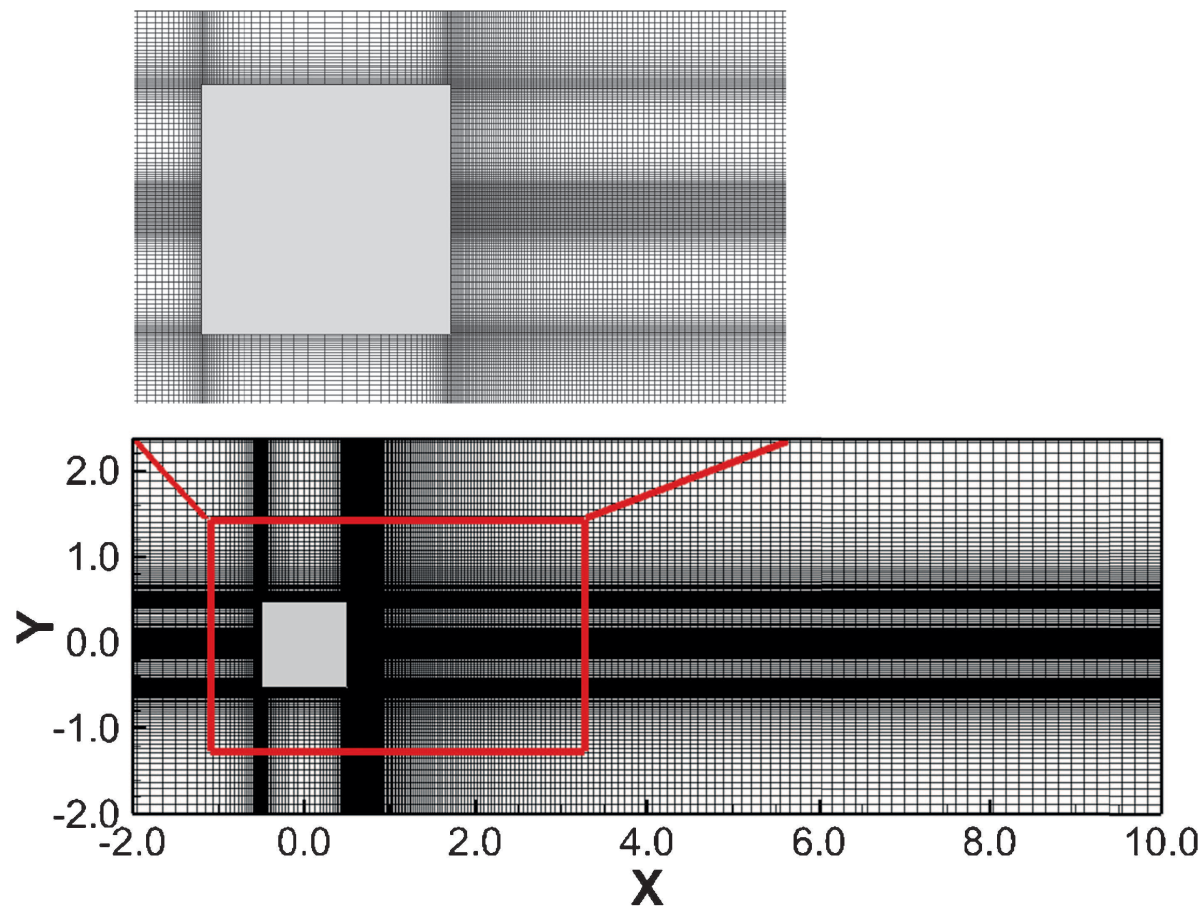

Figure 3. Layout of grids used for the case of a single jet (grid size: $346 \times 294$ ). 


\section{Results and discussion}

As mentioned earlier, the study has been divided into three major cases. The following sections will discuss the results for each case separately. Since all the cases require comparison with the baseline case, the flow past a square cylinder without the presence of any jets has been computed and is presented in figure 4. The instantaneous vorticity contours at any arbitrary time instant (figure 4a) shows clear formation of wake also called Kármán vortex street. The contour plot clearly reveals the alternate shedding of vortices from each side of the cylinder. The repeated rollup of fluid masses are discerned. Similar flow structures have also been experimentally observed by Gerrard (1966) for circular cylinders at Reynolds numbers of about 100. As in figure 4 , the laminar wake remains strongly coherent over a larger distance, while the degree of coherence is expected to be diminished over a smaller distance, at higher Reynolds numbers. The corresponding time-averaged streamlines shown in figure $4 \mathrm{~b}$ illustrates common flow behaviour observed in low Reynolds number steady flow where a pair of bubbles remains attached to the rear face of the cylinder. It is clear from the discussions that the flow in the wake is unsteady, with a dominant periodic component superimposed on the mean velocity field.

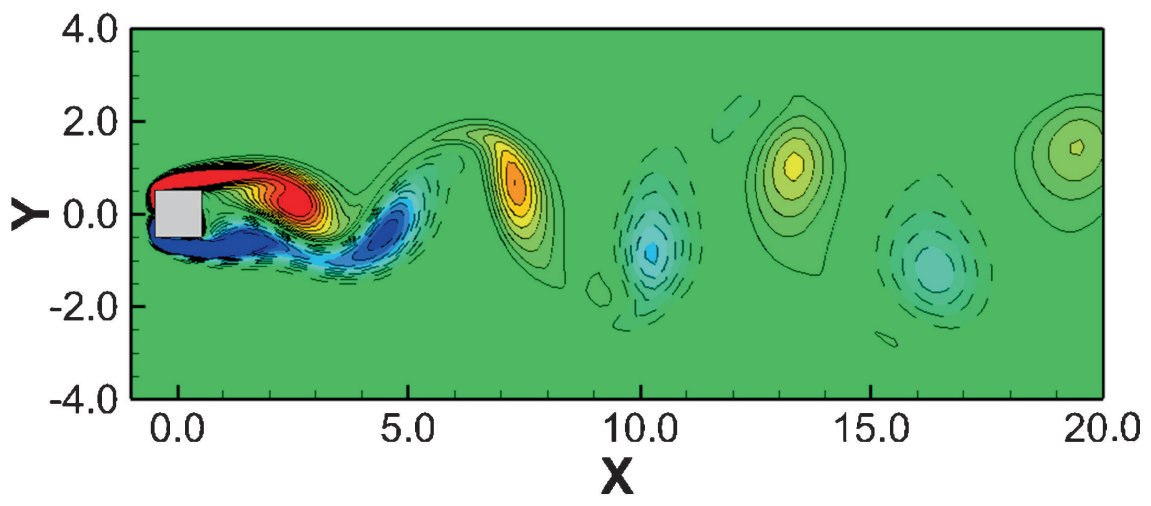

(a)

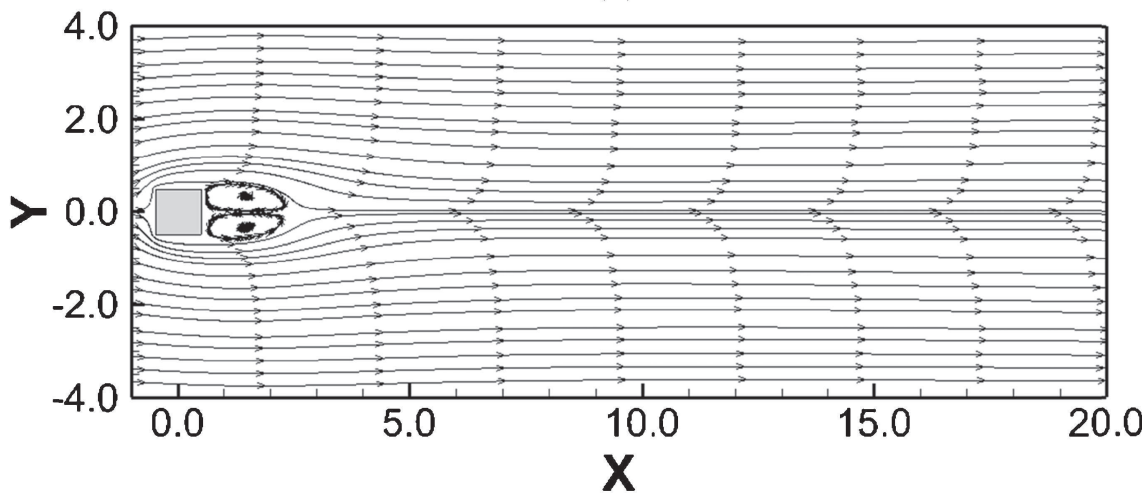

(b)

Figure 4. (a) Instantaneous vorticity contours and (b) time-averaged streamtraces for baseline case. 


\subsection{Flow structure and effect of blowing on vortex shedding}

One may expect that high velocity blowing through a single jet at rear would work similar to that of a case where a splitter plate is attached at the rear face of the cylinder. The splitter plate is found to be helpful in suppressing the vortex shedding by preventing the interaction of the two opposite sign vortices (Saha \& Jaiswal 2011). The velocity profile of the jet for the this analysis is taken to be uniform.

Figure 5a shows the instantaneous vorticity contours for different jet velocities or velocity ratios. It is quite evident from the distribution that as the blowing velocity increases, the shear layer becomes elongated. The undulation of the shear layers has been observed to become lower with increasing jet velocity. At a blowing velocity of 0.87 , the flow does not show any undulations and consequently becomes steady. At lower blowing velocities, the jet does not possess enough momentum to affect the separated shear layers. The interaction of the two separated shear layers is delayed because of the presence of the jet at the center. The jet behaves like an obstruction similar to splitter plate the length of which depends on the jet velocity thus affecting the interaction between two opposite sign separating shear layers. Twin vortices are observed at the rear face of the cylinder because of the presence of jet. Also, because of the induced flow, one additional pair of small bubbles on either side of the twin larger bubbles is seen. The above observations are quite distinct from the streamlines shown in figure 5b. The streamlines give clear evidence of formation of recirculation regions and the flow curvatures. As the blowing velocity
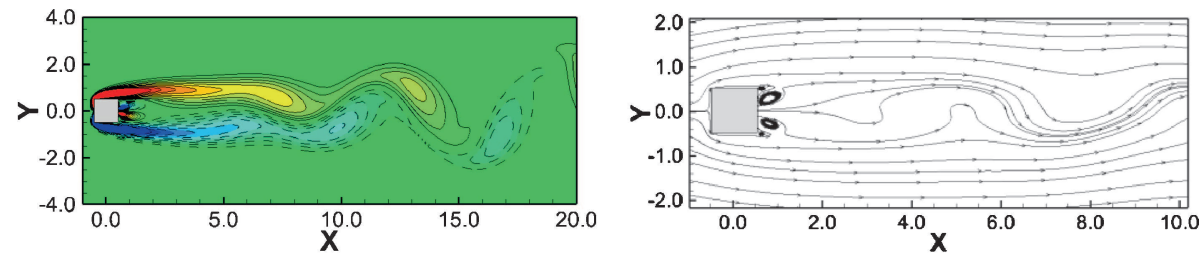

Blowing Velocity 0.50
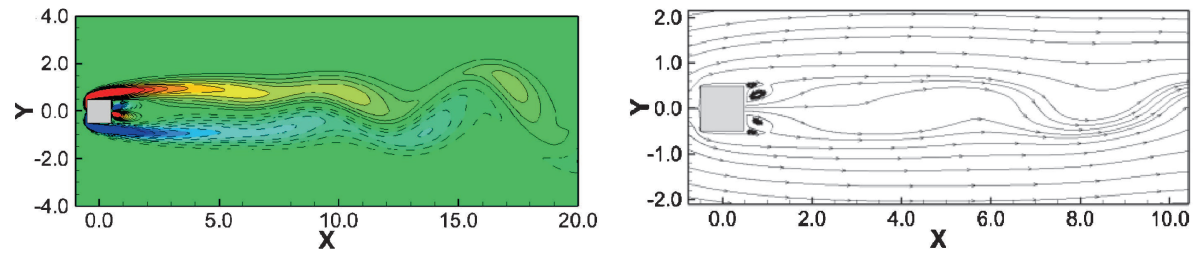

Blowing Velocity 0.70

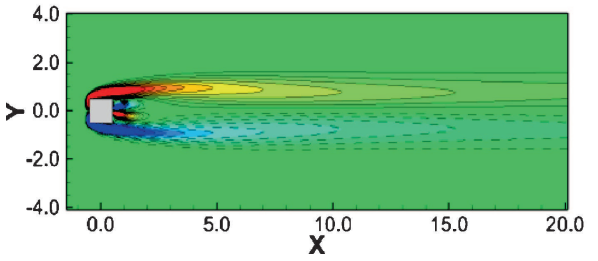

(a)

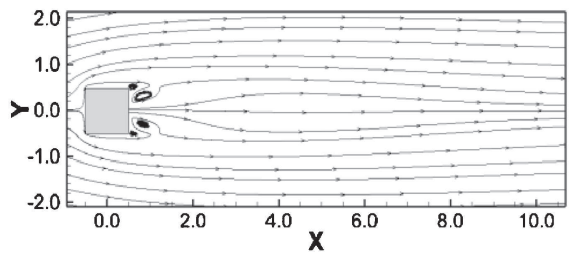

(b)

Blowing Velocity 0.87

Figure 5. Instantaneous (a) vorticity contours and (b) streamlines at different blowing velocities (uniform velocity distribution) for single jet at rear. 
is increased, the jet gradually gains enough momentum and the stream-wise length within which the interaction between the shear layers occurs also increases. Beyond a critical distance, the shear layers lose their strength through viscous diffusion and cannot interact to gain sufficient circulation. Consequently, the tearing of the shear layers and their subsequent shedding becomes impossible.

The time-averaged streamlines for various jet blowing velocities for the single jet case have been shown in figure 6 . As it has been observed, the size and locations of the large twin detached recirculatory bubbles changes with the change of blowing velocity. With increasing blowing velocity, the size shrinks while the location shifts downstream. Finally, at a blowing velocity of 0.87 , the large twin bubble disappears and flow becomes steady without any evidence of vortex shedding. In addition to changes to the large bubbles, the changes in the size and number of attached vortices have also been noticed.

Thus, essentially jet blowing works through two principal mechanisms. At lower range of blowing, it simply shifts the recirculation region away from the cylinder giving rise to partial reduction in fluctuations while at high velocities it also increases the recirculation length (Wood 1967) to a great extent. This fact is testified by figure 7 in which as the jet velocity approaches the critical value at which complete suppression is attained (here 0.87 ) the stream-wise velocity variation along the wake centerline becomes flatter and takes longer distance over which the velocity recovers to positive value though the backflow is delayed. An increase in jet velocity decreases the recovery of stream-wise velocity and is clear from figure 7 . Near the critical values, the recovered velocity becomes asymptotic and is equal to around 0.45 . This is due to the fact that the decrease in unsteadiness causes the entrainment in the wake to be lower. The increase in recirculation length is caused due to increase in the length of the shear layers before they roll up to form vortices. This leads to diffusion of vorticity from the shear layers resulting in a lower strength of main vortices (Gerrard 1966) which are unable to undergo shedding.

\subsection{Effect of jet exit velocity profile}

The jet penetration is expected to depend on the maximum velocity of the jet. Therefore, the jet velocity profile may affect the shedding of vortices by altering the near wake of the flow.

Both uniform and parabolic velocity profiles are chosen for this analysis. Table 1 lists the various cases having different velocity profiles and jet velocities. It is obvious from table 1 that the case with parabolic velocity profile at the jet exit requires considerably lower volume flux to achieve almost similar percentage reduction in Strouhal number in comparison to the uniform profile case. This can be more explicitly confirmed from the fact that complete suppression occurs in parabolic and uniform velocity cases at a volume flux of 0.120 and 0.174 , respectively. The total reduction of $31 \%$ is achieved when parabolic velocity profile jet is used.

A parabolic jet provides the advantage of a higher maximum velocity (keeping the average velocity same as in the uniform profile case) at the centre of the jet. Thus, with the same volume flux as the uniform case the jet penetrates further deep in comparison to uniform profile. Consequently, the interaction between the two opposite sign shear layers is delayed. The centerline variation of axial velocity for parabolic profile has been shown in figure 8 . Unlike the uniform jet velocity profile, the flow with parabolic jet velocity profile shows negligible backflow along the wake centerline. However, similar to uniform velocity, the recovery of stream-wise velocity decreases with increasing jet velocity and attains an asymptotic value of 0.45 .

Apart from the effect of velocity profiles on shedding frequency, mean drag coefficient, $\mathrm{C}_{\mathrm{D} \text {,MEAN }}$ and rms value of drag coefficient, $\mathrm{C}_{\mathrm{D} \text {, RMS }}$ have also been calculated from the flow 


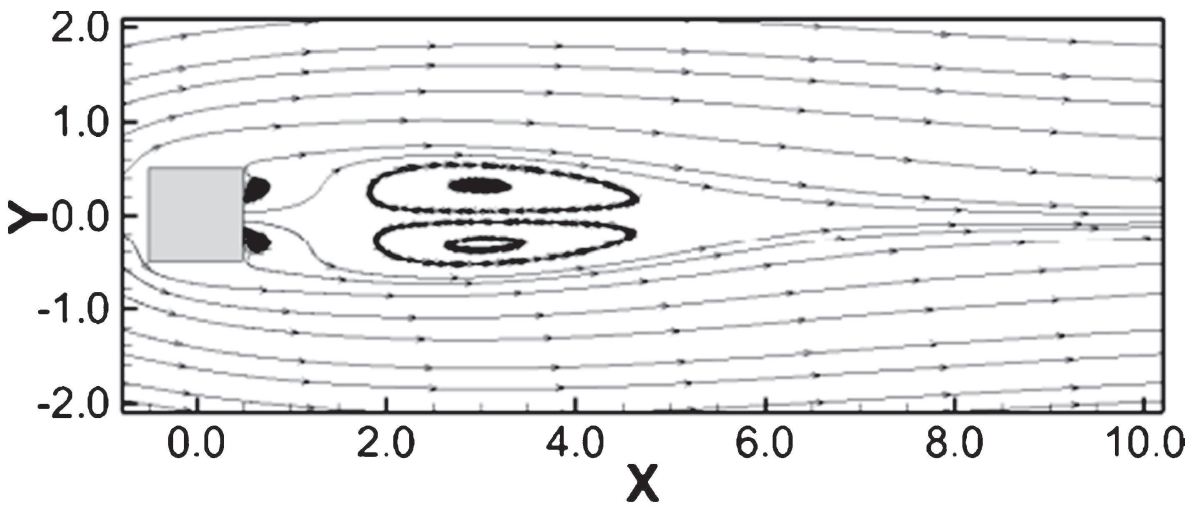

(a) Blowing Velocity 0.50

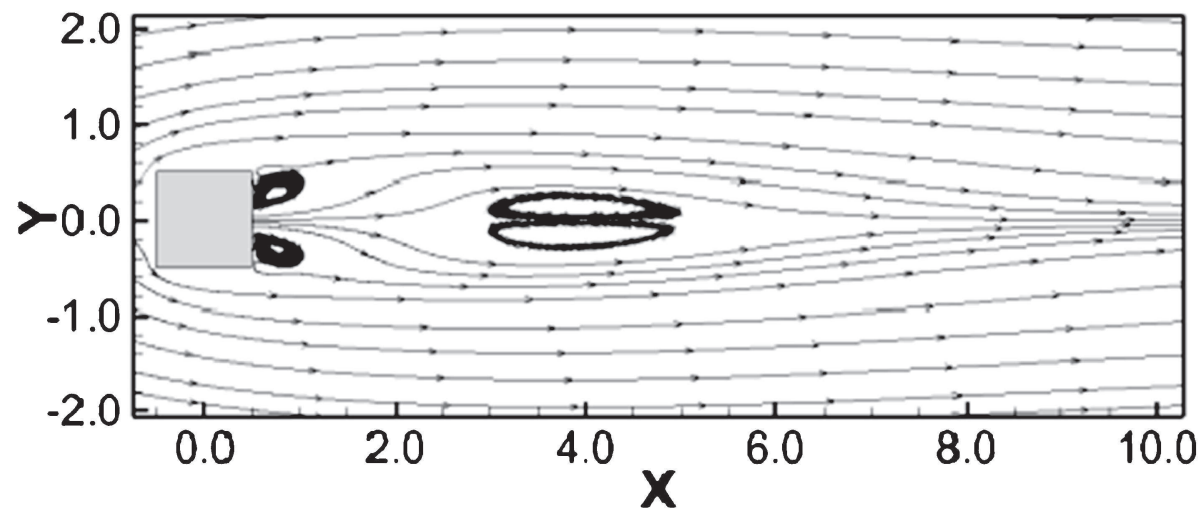

(b) Blowing Velocity 0.70

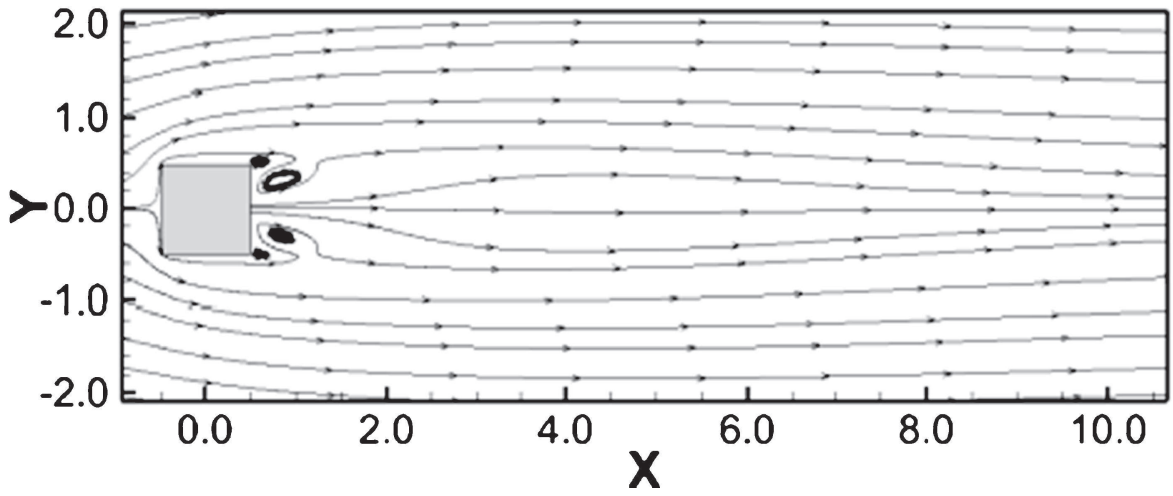

(c) Blowing Velocity 0.87

Figure 6. Time averaged streamtraces at different blowing velocities (uniform velocity distribution) for single jet at rear. 


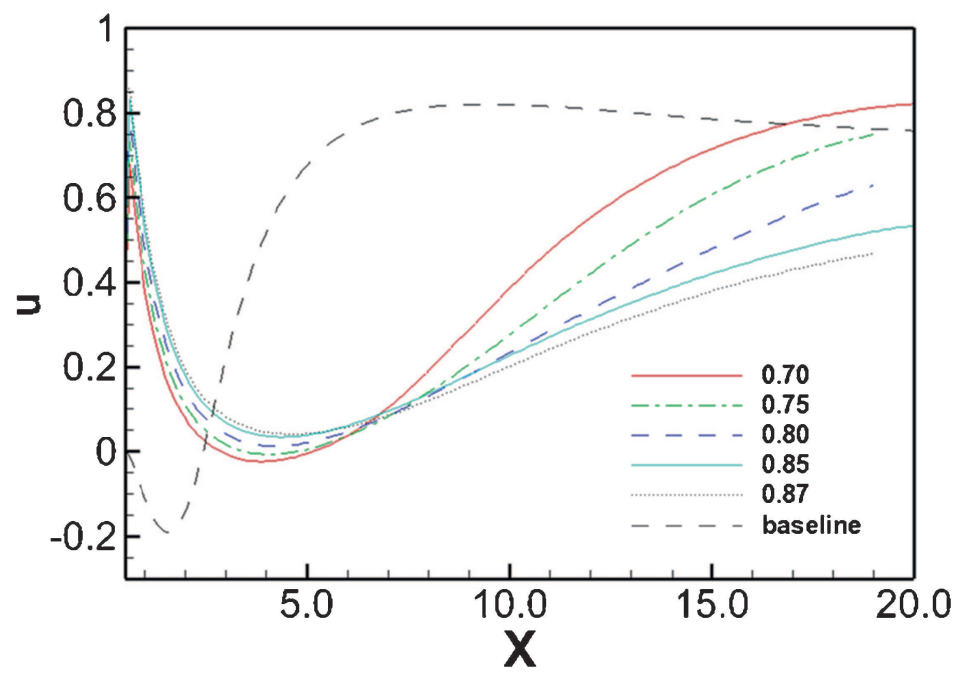

Figure 7. Stream-wise variation of axial velocity for various blowing velocities (uniform velocity distribution) through single jet at rear.

field obtained for both the jet profiles. Table 2 presents the two values for two jet velocity profiles at various jet velocities. The results demonstrate that if the effect of momentum of the jet is neglected in calculating $\mathrm{C}_{\mathrm{D}, \mathrm{MEAN}}$ there is a consistent increase in the drag with the increase in blowing velocity, which is also in consistent with the findings of Kim et al (2003). Nevertheless, there is a drastic reduction in the drag values when the effect of momentum of jet is taken into consideration. It can also be observed quite clearly that the reduction in drag at complete suppression is more in case of uniform velocity profile than the parabolic case.

\subsection{Effect of change in the number of jets}

Numerous simulations were performed for three more cases in addition to the case of single jet with an objective to understand the outcome of the number of jets on the shedding characteristics.

Table 1. Comparison of Strouhal number for various jet blowing velocity for one-jet having different velocity profiles.

\begin{tabular}{lccccccc}
\hline & & \multicolumn{2}{c}{ Strouhal number } & & \multicolumn{2}{c}{ \% reduction in Strouhal number } \\
\cline { 3 - 4 } Sl. no. & $\begin{array}{c}\text { Blowing } \\
\text { velocity }\end{array}$ & $\begin{array}{c}\text { Uniform } \\
\text { velocity profile }\end{array}$ & $\begin{array}{c}\text { Parabolic } \\
\text { velocity profile }\end{array}$ & Volume flux & $\begin{array}{c}\text { Uniform } \\
\text { velocity profile }\end{array}$ & $\begin{array}{c}\text { Parabolic } \\
\text { velocity profile }\end{array}$ \\
\hline 1 & 0.00 & 0.163 & 0.163 & - & - & - \\
2 & 0.50 & - & 0.128 & 0.100 & - & 21.53 \\
3 & 0.55 & - & 0.121 & 0.110 & - & 25.52 \\
4 & 0.57 & - & 0.117 & 0.116 & - & 28.22 \\
5 & 0.60 & - & 0.000 & 0.120 & - & 100.0 \\
6 & 0.70 & 0.126 & 0.000 & 0.140 & 22.82 & 100.0 \\
7 & 0.75 & 0.123 & 0.000 & 0.150 & 24.79 & 100.0 \\
8 & 0.85 & 0.118 & 0.000 & 0.170 & 27.55 & 100.0 \\
9 & 0.87 & 0.000 & 0.000 & 0.174 & 100.0 & 100.0 \\
\hline
\end{tabular}




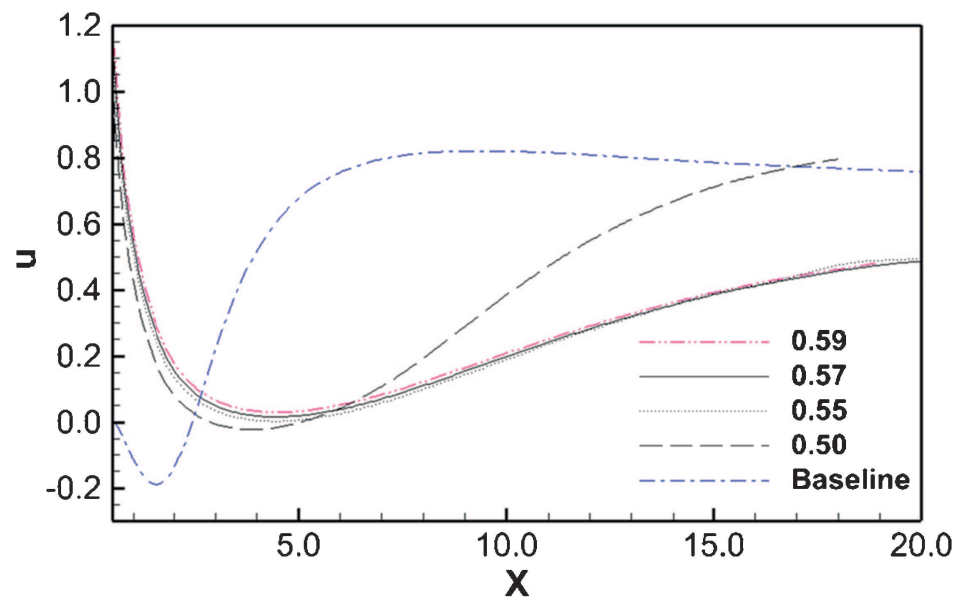

Figure 8. Stream-wise variation of axial velocity for blowing velocities (parabolic velocity distribution) through single jet at rear.

The area exposed to the jets at the rear face is kept constant at $20 \%$ and jets are distributed perfectly symmetrically about the centerline of the square cylinder. As evident from above discussion where it has been shown that the parabolic profile is more effective than uniform profile in suppressing vortex shedding, two-jets, three-jets and four-jets cases are simulated using a parabolic profile at the jet exit.

Complete suppression was attained at a blowing velocity of 0.75 for two-jets case, 0.80 in three-jets case and 1.05 in four-jets case which are all greater than the velocity (0.60) at which suppression was attained with a single jet. The Strouhal number variation with different blowing velocities for all the three cases is illustrated in table 3 . A comparison of the time-averaged flow obtained in the three cases at a constant volume flux of 0.120 is depicted in figure 9. Table 3 shows that an increase in number of jet deteriorates the suppression of vortex shedding. The probable reason for such a behaviour is due to the complex interactions between two adjacent jets which are quite close resulting into a combined jet with lower axial momentum. Consequently, jet penetration suffers and cannot have effective control.

Table 2. Comparison of drag coefficient for various jet blowing velocity for one-jet having different velocity profiles.

\begin{tabular}{|c|c|c|c|c|c|c|}
\hline \multirow[b]{2}{*}{ Sl. no. } & \multirow[b]{2}{*}{$\begin{array}{l}\text { Blowing } \\
\text { velocity }\end{array}$} & \multicolumn{2}{|c|}{$\mathrm{C}_{\mathrm{D}, \text { Mean }}$ (uniform profile) } & \multicolumn{2}{|c|}{$\mathrm{C}_{\mathrm{D}, \text { Mean }}$ (parabolic profile) } & \multirow[b]{2}{*}{$\mathrm{C}_{\mathrm{D}, \mathrm{RMS}}$} \\
\hline & & $\begin{array}{l}\text { Neglecting } \\
\text { effect of Jet }\end{array}$ & $\begin{array}{l}\text { Including } \\
\text { effect of jet }\end{array}$ & $\begin{array}{l}\text { Neglecting } \\
\text { effect of Jet }\end{array}$ & $\begin{array}{l}\text { Including } \\
\text { effect of jet }\end{array}$ & \\
\hline 1 & 0.00 & 1.524 & 1.524 & 1.524 & 1.524 & $5.68 \mathrm{E}-03$ \\
\hline 2 & 0.50 & - & - & 1.502 & 1.000 & $9.52 \mathrm{E}-05$ \\
\hline 3 & 0.55 & - & - & 1.501 & 0.896 & $7.13 \mathrm{E}-05$ \\
\hline 4 & 0.57 & - & - & 1.510 & 0.860 & $2.21 \mathrm{E}-05$ \\
\hline 5 & 0.60 & - & - & 1.523 & 0.803 & $6.94 \mathrm{E}-08$ \\
\hline 6 & 0.70 & 1.554 & 0.574 & - & - & $8.90 \mathrm{E}-05$ \\
\hline 7 & 0.75 & 1.561 & 0.436 & - & - & $6.28 \mathrm{E}-05$ \\
\hline 8 & 0.85 & 1.592 & 0.147 & - & - & $4.62 \mathrm{E}-05$ \\
\hline 9 & 0.87 & 1.599 & 0.086 & - & - & $2.21 \mathrm{E}-06$ \\
\hline
\end{tabular}


Table 3. Comparison of Strouhal number for various jet blowing velocity for various numbers of jets.

\begin{tabular}{lcccccccc}
\hline & Blowing & \multirow{2}{*}{$\begin{array}{c}\text { Volume } \\
\text { Sl. no. }\end{array}$} & \multicolumn{3}{c}{ Strouhal number } & \multicolumn{3}{c}{ \% reduction in Strouhal number } \\
\cline { 4 - 9 } & velocity & flux & Two-jets & Three-jets & Four-jets & Two-jets & Three-jets & Four-jets \\
\hline 1 & 0.00 & - & 0.163 & 0.163 & 0.163 & - & - & - \\
2 & 0.60 & 0.12 & 0.126 & 0.128 & 0.129 & 22.76 & 21.60 & 20.53 \\
3 & 0.65 & 0.13 & 0.123 & - & - & 24.36 & - & - \\
4 & 0.70 & 0.14 & 0.122 & 0.123 & - & 25.46 & 24.66 & - \\
5 & 0.75 & 0.15 & 0.000 & - & 0.120 & 100.00 & - & 26.14 \\
6 & 0.80 & 0.16 & - & 0.118 & - & - & 27.61 & - \\
7 & 0.85 & 0.17 & - & 0.000 & - & - & - & - \\
8 & 0.90 & 0.18 & - & - & 0.114 & - & - & 29.48 \\
9 & 1.05 & 0.21 & - & - & 0.000 & - & - & 100.0 \\
\hline
\end{tabular}

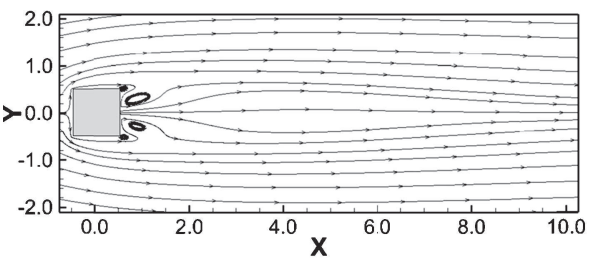

(a) one-jet

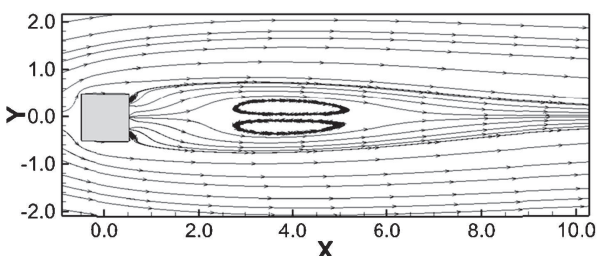

(c) three-jets

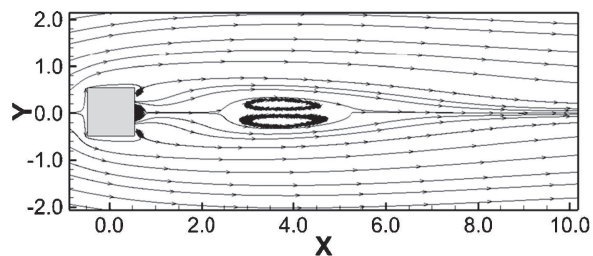

(b) two-jets

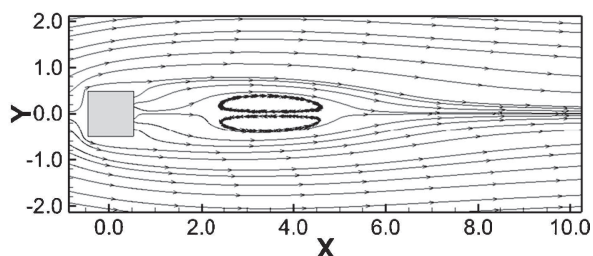

(d) four-jets

Figure 9. Time averaged streamlines for various numbers of jets at a volume flux of 0.120.

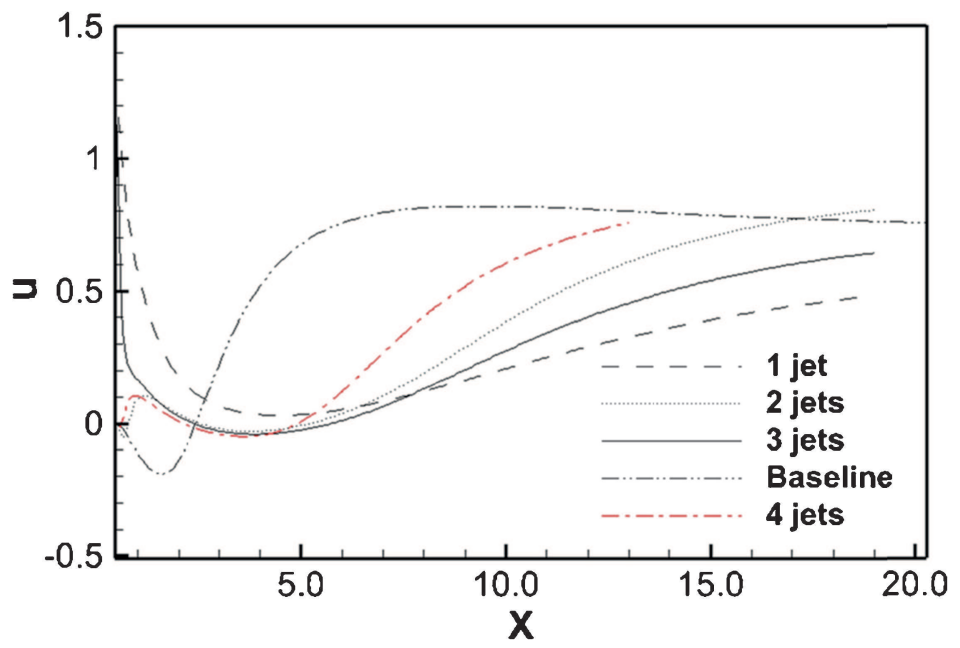

Figure 10. Stream-wise variation of axial velocity along wake centreline for different numbers of jets with parabolic profile at jet exit and constant volume flux of 0.120 . 
Figure 10 shows the wake centerline variation of stream-wise velocity in all three cases at a constant volume flux of 0.120 . It can be observed from figure 10 that the interaction of the separated shear layers is delayed as the number of jets decreases and is clear from the lowest recovery of stream-wise velocity for one-jet case because of lower entrainment of fluid into the wake. Although, the recovery rate of velocity becomes higher with increasing number of jets no significant changes in the mean velocity distribution has been observed for two-, three- and four-jets cases. This may be due to the complex interaction among the adjacent jets causing loss of their effectiveness.

\section{Conclusions}

The present study is aimed at understanding of the effect of jet blowing on vortex shedding characteristics. The numerical simulations reveal that blowing suppresses vortex shedding by lowering the interaction of separated shear layers leading to weak shear layers due to vorticity diffusion. Complete suppression with single jet having uniform jet exit velocity profile was achieved at a mass flux of 0.120 and a blowing velocity of 0.87 . Furthermore, parabolic jet is found to be more effective than its uniform counterpart. A remarkable reduction in drag coefficient is achieved when the effect of jet momentum is taken into consideration. The asymptotic recovered stream-wise velocity is observed to be around 0.45 irrespective of the jet velocity profile. Finally, a comparative study of the effect of number of jets on shedding reveals that increasing the number of jets, deteriorates the performance of control. Complete suppression with two-jets occurs at a volume flux of 0.150 , while the corresponding values for three-jets and four-jets are 0.170 and 0.210 , respectively.

\section{Acknowledgement}

One of the authors (Ankit Shrivastava) would like to thank Summer Undergraduate Research Grant for Excellence 2013, Indian Institute of Technology Kanpur for providing him the platform and financial help to carry out this research work.

\section{Nomenclature}

D Height and Width of the square cylinder (non-dimensional)

$\mathrm{L}_{\mathrm{u}} \quad$ Upstream dimension of the flow domain (non-dimensional)

$\mathrm{L}_{\mathrm{d}} \quad$ Downstream dimension of the flow domain (non-dimensional)

$\mathrm{H} \quad$ Transverse dimension of the flow domain (non-dimensional)

$\mathrm{C}_{\mathrm{D}, \mathrm{MEAN}} \quad$ Mean drag coefficient

$\mathrm{C}_{\mathrm{D}, \mathrm{RMS}} \quad \mathrm{RMS}$ drag coefficient

$f \quad$ Frequency of vortex shedding, $\mathrm{Hz}$

$p \quad$ Static Pressure (non-dimensional)

Re Reynolds number, $u_{\mathrm{av}}^{*} \mathrm{D}^{*} / v$

St Strouhal number, $f \mathrm{D}^{*} / u_{\mathrm{av}}^{*}$

$t \quad$ Time (non-dimensional)

$u, v \quad$ Stream-wise and transverse velocity components (non-dimensional)

$x, y \quad$ Stream-wise and transverse coordinates (non-dimensional) 
Greek symbols

$v$

Kinematic viscosity, $\mathrm{m}^{2} / \mathrm{s}$

$\rho$

Density, $\mathrm{kg} / \mathrm{m}^{3}$

\section{Subscripts}

av

RMS

c

u

d

* quantities are dimensional ones.

Average

Root mean square

Celerity constant related

Upstream

Downstream

\section{References}

Akansu Y E and Firhat E 2010 Control of flow around a square prism by slot injection from the rear surface. Experimental Thermal and Fluid Sci. 34: 906-914

Arcas D R and Redekopp L G 2004 Aspects of wake vortex control through base blowing/suction. Phys. Fluids 16: 452-456

Biringen S 1984 Active Control of transition by periodic blowing and suction. Phys. Fluids 27: 1345-1347

Blackburn H and Henderson R 1999 A study of two-dimensional flow past an oscillating cylinder. J. Fluid Mech. 385: 255-286

Cetiner O and Rockwell D 2003 Controlled Oscillation of a cylinder: A new wake state. J. Fluids Struct. 17: 337-343

Choi J 2006 Mechanism of drag reduction by surface modification on sphere: Dimples, roughness and trip wire: $\mathrm{PhD}$. Thesis, Seoul national University, Korea

Choi H, Jeon W P and Kim J 2008 Control of flow over a bluff body. Ann. Rev. Fluid Mech. 40: 113-139

Cohen R D 1991 Predicting the effects of surface suction and blowing on the strouhal frequencies in Vortex Shedding. JSME Int. J. Series II 34(1): 30-38

Cuhadaroglu B, Akansu Y E and Turhal A O 2007 An experimental study on the effects of uniform injection through one perforated surface of a square cylinder on some aerodynamic parameters. Experimental Thermal and Fluid Sci. 31: 909-915

Dong S, Triantafyllou G S and Karniadakis G E 2008 Elimination of vortex street in bluff flows. Physical Review Lett. Article No.204501

Fransson J H M, Konieczny P and Alfredson P H 2004 Flow around a porous cylinder subject to continuous suction or blowing. J. Fluids Struct. 19: 1031-1048

Gad-el-Haq M 2000 Flow control, passive, active and reactive flows, First ed.,. London: Cambridge University Press

Gerrard J H 1966 The mechanics of formation region of vortices behind bluff bodies. J. Fluid Mech. 25: 401-413

Harlow F H and Welch J E 1965 Numerical calculation of time-dependent viscous incompressible flow fluid with free surfaces. Phys. Fluids 8: 2182-2188

Kim S and Lee C 2000 Investigation of flow around circular cylinder under the influence of electromagnetic force. Exp. Fluids 28: 252-260

Kim D H, Yang K S and Eom J S 2003 Confined vortex shedding past a square cylinder with planar jet. JSME Int. J. Series B 46(2): 316-325

Kumar A Raghavan, Sohn Chan-Hyun and Gowda H L 2008 Passive control of vortex induced vibrations: An overview. Recent Patents on Mechanical Eng. 1: 1-11

Kwon K and Choi H 1996 Control of laminar vortex shedding behind circular cylinder using splitter plates. Phys. Fluids 8: 479-486 
Lankadasu A and Vengadesan S 2008 Onset of Vortex shedding in planar shear flow past a square cylinder. Int. J. Heat and Fluid Flow 29: 1054-1059

Lee S and Kim H 1997 The effect of surface protrusions on the near wake of a circular cylinder. J. Wind Eng. Ind. Aerodyn. 69-71: 351-361

Ling Lisa Mei 1992 Numerical analysis on strouhal frequencies in vortex shedding over square cylinders with surface suction and blowing. PhD Thesis, Rice University, U.S

Mathelin L, Bataille F and Lallemand A 2002 The effect of Uniform Blowing on the flow past a circular cylinder. J. Fluids Eng. 124(2): 452-464

Orlanski I 1976 A simple Boundary condition for unbounded flows. J. Comput. Phys. 21: 251-269

Saha A K 2013 Unsteady flow past a finite square cylinder mounted on a wall at low Reynolds number. Comp. Fluids 88(15): 599-615

Saha A K and Jaiswal R 2011 Control of vortex shedding past a square cylinder using splitter plate at low Reynolds number, Proceeding of the 38th National on Fluid Mechanics and Fluid Power, December 15-17, 2011, Maulana Azad National Institute of Technology, Bhopal, India

Saha A K, Muralidhar K and Biswas G 2000 Transition and chaos in two-dimensional flow past a square cylinder. J. Eng. Mech. 126: 523-532

Sen S, Mittal S and Biswas G 2011 Flow past a square cylinder at low Reynolds numbers. Int. J. Numer. Meth. Fluids 67: 1160-1174

Strykowski P J and Sreenivansan K R 1990 On the formation and suppression of vortex shedding at low Reynolds number. J. Fluid Mech. 218: 71-107

Wood C J 1967 Visualization of an incompressible wake with base bleed. J. Fluid Mech. 29: 259-273

Zdravkovich M M 1981 Review and classification of various aerodynamic and hydrodynamic means for suppressing vortex shedding. J. Wind Eng. Industrial Aerodynamics 7: 145-189 